\title{
DOSES DE NITROGÊNIO, FÓSFORO E POTÁSSIO NA NUTRIÇÃO DO PORTA- ENXERTO CÍTRICO DE LIMOEIRO 'CRAVO'
}

\section{NITROGEN, PHOSPRORUS AND POTASSIUM LEVELS IN NUTRITION OF CITRUS ROOTSTOCK 'RANGPUR' LIME}

\author{
Diego Wyllyam do VALE 1 \\ Renato de Mello PRADO² \\ Henrique Antunes de SOUZA ${ }^{3}$ \\ Antônio Baldo Geraldo MARTINS ${ }^{4}$
}

\begin{abstract}
RESUMO
Neste trabalho avaliou-se o efeito da aplicação de diferentes doses de N, P e K sobre o estado nutricional do portaenxerto cítrico limoeiro 'Cravo'. O experimento foi realizado na Faculdade de Ciências Agrárias e Veterinárias - Unesp, sob delineamento experimental em blocos casualizados, em esquema fatorial $3^{3}+1$, com três repetições. As doses padrões de $N$, $\mathrm{P}$ e K foram de $920 \mathrm{mg} \mathrm{dm}^{-3}, 100 \mathrm{mg} \mathrm{dm}^{-3} \mathrm{e} 790 \mathrm{mg} \mathrm{dm}^{-3}$, respectivamente. Para compor os tratamentos utilizou-se: $\mathrm{D}_{1}-$ metade da dose padrão, $D_{2}-$ a dose padrão e $D_{3}$ o dobro da dose padrão, além da testemunha (sem adubação). Após a emergência das plântulas, as adubações foram realizadas via fertirrigação, aumentando a concentração dos nutrientes na solução de acordo com o crescimento das mudas. As adubações $\mathrm{N}$ e K, em cada unidade experimental, foram realizadas durante quinze semanas. Aos 120 dias após a emergência das plântulas foi realizada a avaliação dos teores de nutrientes na massa seca total e o acúmulo de $\mathrm{N}, \mathrm{P}$ e K nas plantas. A dose de nitrogênio de $1.840 \mathrm{mg} \mathrm{dm}^{-3}$ proporcionou maior teor e acúmulo de $\mathrm{N}$, para o intervalo de doses aplicado. A aplicação de $\mathrm{P}$ e K acima de 100 e $790 \mathrm{mg} \mathrm{dm}^{-3}$ não proporcionou aumento na absorção desses nutrientes, respectivamente.
\end{abstract}

Palavras-chave: Citrus limonia L.; adubação; fertirrigação; acúmulo; teor.

\section{ABSTRACT}

The objective of this work was to evaluate the effects of doses a fertilizer N, P and $\mathrm{K}$ on the nutritional status of citrus rootstock of 'Rangpur' lime, in UNESP/FCAV, Brazil. The experimental design was randomized blocks, in a factorial experimental design in $3^{3}+1$, with three replicates. The standard doses of $\mathrm{N}, \mathrm{P}$ and $\mathrm{K}$ were $920 \mathrm{mg} \mathrm{dm}^{-3}, 100 \mathrm{mg} \mathrm{dm}^{-3} \mathrm{and}_{790}$ $\mathrm{mg} \mathrm{dm}^{-3}$, respectively. The treatments were: D1 - half the standard dose, D2 - the dose standard, and D3 - double the standard dose given, beyond the control (without fertilizer). After the emergence of the seedlings, fertilizations were done though fertirrigation. The concentration of nutrients in solution was to increase to the growth of the seedlings. The fertilizations $\mathrm{N}$ and $\mathrm{K}$, in each experimental unit, were applied until fifteen weeks. Already the phosphates fertilizations were built into the substrate at the time of sowing. After 120 days the emergence, the seedlings were evaluated at nutritional status. From the results of dry matter and nutrient content calculated is the accumulation of $\mathrm{N}, \mathrm{P}$ and $\mathrm{K}$ in plants. The nitrogen level $1,840 \mathrm{mg}$ $\mathrm{dm}^{-3}$ content and a higher accumulation of $\mathrm{N}$, for the range of doses studied. The increase of $\mathrm{P}$ and $\mathrm{K}$ above of 100 and 790 $\mathrm{mg} \mathrm{dm}^{-3}$ not provide increase in the absorption of these nutrients, respectively.

Key-words: Citrus limonia L.; fertilization, fertirrigation; accumulation; content.

\footnotetext{
1 Engenheiro Agrônomo, Mestrando em Produção Vegetal. Departamento de Solos e Adubos. Faculdade de Ciências Agrárias e Veterinárias, Universidade Estadual Paulista Júlio de Mesquita Filho (UNESP), Campus de Jaboticabal. Via de Acesso Professor Paulo Donato Castellane, s/nº, 14884-900, Jaboticabal, São Paulo - Brasil. E-mail: diegodwv@yahoo.com.br. Autor para correspondência.

${ }^{2}$ Engenheiro Agrônomo, Doutor em Agronomia, Professor do Departamento de Solos e Adubos. Faculdade de Ciências Agrárias e Veterinárias, Universidade Estadual Paulista Júlio de Mesquita Filho, Campus de Jaboticabal. E-mail: rmprado@pq.cnpq.br.

${ }^{3}$ Engenheiro Agrônomo, Mestrando em Produção Vegetal. Departamento de Solos e Adubos. Faculdade de Ciências Agrárias e Veterinárias, Universidade Estadual Paulista Júlio de Mesquita Filho, Campus de Jaboticabal. Jaboticabal, São Paulo - Brasil. E-mail: henrique.antuness@yahoo.com.br.

${ }^{4}$ Engenheiro Agrônomo, Doutor em Agronomia, Professor do Departamento de Produção Vegetal. Faculdade de Ciências Agrárias e Veterinárias, Universidade Estadual Paulista Júlio de Mesquita Filho, Campus de Jaboticabal. Jaboticabal, São Paulo - Brasil. E-mail: baldo@fcav.unesp.br.
} 
VALE, D.W. et al. Doses de nitrogênio, fósforo e potássio...

\section{INTRODUÇÃO}

Estima-se que o Brasil cultive cerca de 803 mil ha com plantas cítricas, totalizando uma produção aproximada de 438 milhões de toneladas de frutos e 1.402.000 toneladas métricas de suco de laranja concentrado (SLCC), fato este que the confere o título de maior produtor mundial de SLCC (AGRIANUAL, 2007).

Em culturas perenes, como a do Citrus, a precocidade para o início da produção é importante para garantir retorno econômico mais rápido do investimento feito na instalação do pomar. Para isto, a muda cítrica com qualidade e com adequado estado nutricional torna-se o insumo mais importante no sucesso da formação de um pomar com alta homogeneidade e vigor, garantindo um adequado desenvolvimento das plantas (Lima, 1986).

O porta-enxerto pode influenciar o desenvolvimento dos frutos da variedade copa, e a maior parte dessa influência deve-se à capacidade de absorção de água e nutrientes pela planta (Castle, 1995). O porta-enxerto cítrico também pode influenciar no conteúdo mineral da folha (Dasberg, 1996) e na eficiência de produção dos frutos (Quaggio et al., 2004).

Estima-se que no Brasil na década de 90, cerca de $80 \%$ dos pomares eram constituídos pelo porta-enxerto limoeiro 'Cravo' (Citrus limonia Osbeck.), pela sua característica de induzir vigor, maior tolerância ao estresse hídrico e alta produtividade as plantas (Schafer et al., 2006).

A eficiência da aplicação de fertilizantes tem papel fundamental na produção de mudas cítricas. $\mathrm{O}$ nitrogênio $(\mathrm{N})$ é considerado o nutriente mais importante nos programas de adubação e torna-se especialmente crítico para a produção de mudas cítricas, em que a densidade de plantas é elevada, com rápido crescimento vegetativo (Esposti \& Siqueira, 2004).

Existem indicações de respostas positivas de porta-enxertos cítricos cultivados em tubetes à adubação com $\mathrm{N}$, nos primeiros quatro meses após a semeadura (Carvalho \& Souza, 1996; Decarlos Neto et al., 2002) e, também, à aplicação de N, fósforo $(\mathrm{P})$ e potássio $(\mathrm{K})$ durante toda a fase de formação das mudas (Bernardi et al., 2000).

Além da dose adequada, é importante o conhecimento da freqüência de adubação, uma vez que pode ocorrer intensa lixiviação de nutrientes, provocada pelas constantes irrigações e devido às pequenas dimensões dos recipientes (Barroso et al., 2000). Assim, há necessidade de que os nutrientes sejam parcelados em cobertura, principalmente as fontes de $\mathrm{N}$ e K.

Uma forma promissora de fornecimento de nutrientes para a produção de mudas é por fertirrigação, uma vez que o nutriente é fornecido juntamente com a água (essencial para sua absorção) apresenta outras vantagens, entre as quais a melhor distribuição do fertilizante e a possibilidade de maior parcelamento das adubações, aumentando a absorção de nutrientes pelas plantas (Duenhas et al., 2005). Entretanto, o número de pesquisas que tratam da aplicação de nutrientes via fertirrigação em mudas é baixo, havendo necessidade de mais informações que possam sustentar uma recomendação de fertirrigação para a produção de mudas de citros, cultivadas em recipientes preenchidos com substrato inerte.

Assim este trabalho teve por objetivo avaliar o efeito da adubação N, P e K no estado nutricional do porta-enxerto de limoeiro 'Cravo'.

\section{MATERIALE MÉTODOS}

O experimento foi realizado na Faculdade de Ciências Agrárias e Veterinárias - UNESP em Jaboticabal, SP $\left(21^{\circ} 15^{\prime} \mathrm{S}, 48^{\circ} 19^{\prime} \mathrm{W}\right.$ e $\left.605 \mathrm{~m}\right)$. O clima da região é do tipo subtropical-mesotérmico, ou seja, com verão úmido e inverno seco. A temperatura anual do local é de $22^{\circ} \mathrm{C}$.

A produção de "seedlings" de limoeiro 'Cravo' (Citrus limonia L. Osbeck) foi realizada em um viveiro telado com tela anti-afídica com abertura de $1 \mathrm{~mm}^{2}$, e com bancadas elevadas a $0,3 \mathrm{~m}$ do solo. As sementes obtidas de uma planta de limoeiro 'Cravo' foram semeadas em 27/12/2005, em tubetes $\left(56 \mathrm{~cm}^{3}\right)$, preenchidos com substrato à base de composto de casca de pinus e vermiculita fina (densidade igual a $418 \mathrm{~kg} \mathrm{~m}^{-3}$ ), cuja caracterização química, realizada segundo método holandês adaptado de Sonneveld \& Elderen (1994), revelou que: $\mathrm{CE}=1,5 \mathrm{dS} \mathrm{m}^{-1} ; \mathrm{pH}=5,9 ; \mathrm{N}$ (nitrato) $=2,4 \mathrm{mg}$ $\mathrm{dm}^{-3} ; \mathrm{N}$ (amônia) $=31,4 \mathrm{mg} \mathrm{dm}^{-3} ; \mathrm{P}=16,3 \mathrm{mg} \mathrm{dm}^{-3} ; \mathrm{K}$ $=67,9 \mathrm{mg} \mathrm{dm}^{-3} ; \mathrm{Ca}=108,9 \mathrm{mg} \mathrm{dm}^{-3} ; \mathrm{Mg}=58,8 \mathrm{mg}$ $\mathrm{dm}^{-3} ; \mathrm{S}=188,7 \mathrm{mg} \mathrm{dm}^{-3} ; \mathrm{Cl}=27,0 \mathrm{mg} \mathrm{dm}^{-3} ; \mathrm{Na}=9,6$ $\mathrm{mg} \mathrm{dm}{ }^{-3} ; \mathrm{B}=0,1 \mathrm{mg} \mathrm{dm}^{-3} ; \mathrm{Cu}=0,1 \mathrm{mg} \mathrm{dm}^{-3} ; \mathrm{Fe}=0,2$ $\mathrm{mg} \mathrm{dm}^{-3} ; \mathrm{Mn}=1,3 \mathrm{mg} \mathrm{dm}^{-3}$ e $\mathrm{Zn}=0,1 \mathrm{mg} \mathrm{dm}^{-3}$.

$O$ delineamento experimental foi blocos casualizados em esquema fatorial $3^{3}+1$, sendo três fatores (NPK) aplicados em três doses, além da testemunha (sem adubação), com três repetições. Cada repetição foi composta por duas plantas (uma por tubete).

As doses padrões de N, P e K adotadas para a produção do porta-enxerto foram de $920 \mathrm{mg}$ $\mathrm{dm}^{-3}$ (Ruschel et al., 2004), $100 \mathrm{mg} \mathrm{dm}^{-3}$ (Boaventura, 2003) e $790 \mathrm{mg} \mathrm{dm}^{-3}$ (Ruschel et al., 2004), respectivamente. Assim, para compor os tratamentos, foram utilizadas: $D_{1}=$ metade da dose padrão; $D_{2}=$ a dose padrão; e, $D_{3}=$ duas vezes a dose padrão, além da testemunha (sem adubação). No momento da semeadura, realizou-se a incorporação do adubo fosfatado ao substrato. Após a emergência das plântulas, as adubações $\mathrm{N}$ e K, em cada unidade experimental, foram realizadas durante as quinze semanas subseqüentes, iniciando-se em 31/01/2006. As adubações de $\mathrm{N}$ e $\mathrm{K}$ foram realizadas via fertirrigação, em que a concentração dos nutrientes na solução foi aumentando de acordo com o crescimento das plantas ao longo de 15 semanas (n), da seguinte forma: $n 1=2 \%$; $2=2 \%$; $3=2 \%$; $4=5 \%$; n5=5\%; 
$n 6=5 \% ; n 7=5 \% ; n 8=8 \% ; n 9=8 \% ; n 10=8 \% ; n 11=10 \%$; $n 12=10 \% ; n 13=10 \% ; n 14=10 \%$ e $n 15=10 \%$. Em cada semana, as doses foram divididas em duas aplicações.

Como fontes de N, P e K foram utilizadas os fertilizantes: nitrato de amônio $\left(340 \mathrm{~g} \mathrm{~kg}^{-1}\right.$ de $\left.\mathrm{N}\right)$, superfosfato triplo $\left(440 \mathrm{~g} \mathrm{~kg}^{-1}\right.$ de $\left.\mathrm{P}_{2} \mathrm{O}_{5}\right)$ e o cloreto de potássio $\left(60 \mathrm{~g} \mathrm{~kg}^{-1}\right.$ de $\left.\mathrm{K}_{2} \mathrm{O}\right)$.

Além das doses de N, P e K, realizaram-se uma aplicação de $0,5 \mathrm{~g}_{\text {planta }}{ }^{-1}$ de sulfato de cálcio com a função de atender as exigências nutricionais da cultura. Os micronutrientes foram fornecidos através de pulverizações mensais nas seguintes doses $\left(\mathrm{g} \mathrm{dm}^{-3}\right)$ : $\mathrm{B}=0,2 ; \mathrm{Mn}=0,5$ e $\mathrm{Zn}=0,6$ (Bernardi et al., 2000). O Fe também foi fornecido via fertirrigação na quantidade de $2 \mathrm{~cm}^{3}$ por tubete da solução na concentração de $0,45 \mathrm{mg} \mathrm{dm}^{-3}$ de $\mathrm{Fe}-$ EDDHA (ácido etilenodiamina - de N, $\mathrm{N}^{1}-[2-$ hidroxifenil acético]).

Aos quatro meses após a emergência das plântulas (17/05/2006), estas foram colhidas, lavadas e colocadas em sacos de papel para secagem em estufa de ventilação forçada de ar, a uma temperatura entre 65 a $70^{\circ} \mathrm{C}$, até atingir massa constante. Assim, determinou-se a massa seca da planta inteira (parte aérea e raízes).
Posteriormente o material vegetal (parte aérea e sistema radicular) foi triturado em moinho (peneira com diâmetro de malha de $1 \mathrm{~mm}$ ) para a determinação dos teores de nutrientes na massa seca total, pelos métodos descritos por Malavolta (1997). A partir dos resultados da massa seca e do teor de nutrientes calculou-se o acúmulo de N, P e K nas plantas (teor $x$ massa seca total).

As análises de variância para avaliar o efeito das doses será aplicado o estudo de regressão polinomial do teor e acúmulo de $\mathrm{N}, \mathrm{P}$ e K utilizando o programa ESTAT (1994) até $5 \%$ de probabilidade e as superfícies de respostas foram realizadas utilizando o programa SAS (SAS INSTITUTE, 1996).

\section{RESULTADOS E DISCUSSÃO}

\section{Teores dos nutrientes}

Foram constatados efeitos da aplicação de $\mathrm{N}$ e $\mathrm{P}$, tendo o primeiro afetado os teores de $\mathrm{N} \mathrm{e} \mathrm{K}$, e o último afetando os teores de $\mathrm{P}$ e K (Tabela 1). Houve interação $\mathrm{N} x \mathrm{P}$ para o teor de $\mathrm{K}$, e a interação de $\mathrm{N} x$ $\mathrm{K}$ para o teor de $\mathrm{N}$ do porta-enxerto. Observou-se o incremento linear no teor de fósforo, com o aumento das doses deste nutriente.

TABELA 1 - Teores de nutrientes na planta inteira do porta-enxerto limoeiro 'Cravo', aos quatro meses após a emergência, em função da adubação $\mathrm{N}, \mathrm{P}$ e $\mathrm{K}^{(1)}$.

\begin{tabular}{lllll}
\hline Variável $^{(2)}$ & \multicolumn{1}{c}{ Equação } & Doses $^{(3)}$ & Teste $^{(1)}$ & $\mathrm{Y} \mathrm{max.}^{(4)}$ \\
\hline T.N. & $\begin{array}{l}\mathrm{Y}=9,206+\left(8,37 \mathrm{e}^{-4 *} \mathrm{~N}\right)-\left(4,88 \mathrm{e}^{-4 *} \mathrm{~K}\right)+\left(2,143 \mathrm{e}^{-6}\right. \\
\left.{ }^{*} \mathrm{~N}^{*} \mathrm{~N}\right)-\left(1,078 \mathrm{e}^{-6 *} \mathrm{~N}^{*} \mathrm{~K}\right)+\left(7,467 \mathrm{e}^{-7 *} \mathrm{~K}^{*} \mathrm{~K}\right)\end{array}$ & $\begin{array}{l}\mathrm{N}=1.840 \mathrm{mg} \mathrm{dm}^{-3} \\
\mathrm{~K}=395 \mathrm{mg} \mathrm{dm}^{-3}\end{array}$ & $11,20^{*}$ & 17,14 \\
\hline T.P. & $\mathrm{Y}=(0,0013 \mathrm{P})+1,35$ & $\mathrm{P}=200 \mathrm{mg} \mathrm{dm}^{-3}$ & $15,32^{* *}$ & 1,61 \\
\hline T.K. & $\mathrm{Y}=11,799+\left(0,003^{*} \mathrm{~N}\right)-\left(6,482 \mathrm{e}^{-4 *} \mathrm{P}\right)-\left(1,304 \mathrm{e}^{-6}\right.$ & $\mathrm{P}=65 \mathrm{mg} \mathrm{dm}^{-3}$ & $11,10^{*}$ & 13,76 \\
\hline
\end{tabular}

(1)** - Significativo a $1 \%$ de probabilidade; * - significativo a $5 \%$ de probabilidade; ${ }^{\text {ns }}$ não significativo a $5 \%$ de probabilidade;

(2) T.N. - teor de nitrogênio $\left(\mathrm{g} \mathrm{kg}^{-1}\right)$; T.P. - teor de fósforo $\left(\mathrm{g} \mathrm{kg}^{-1}\right)$; T.K. - teor de potássio $\left(\mathrm{g} \mathrm{kg}^{-1}\right)$;

${ }^{(3)}$ Doses - dose do fertilizante ( $\mathrm{mg} \mathrm{dm}^{-3}$ de substrato) responsável pelo maior teor do nutriente;

(4) $\mathrm{Y}$ max. - maior teor do nutriente $\left(\mathrm{g} \mathrm{kg}^{-1}\right)$.

Verifica-se que as doses de $\mathrm{N}$ igual a 1.840 $\mathrm{mg} \mathrm{dm}{ }^{-3}$ e de $\mathrm{K}$ igual a $395 \mathrm{mg} \mathrm{dm}^{-3}$ foram as responsáveis pelo maior teor de nitrogênio para o intervalo de doses estudado. Pode-se constatar que a maior dose de $\mathrm{N}$ foi responsável pelo maior teor foliar de $\mathrm{N}$, resultado semelhante ao constatado por Carvalho et al. (2000), entretanto estes autores observaram um efeito quadrático nesta relação. Serrano et al. (2004) verificaram resposta linear e crescente para os teores de $\mathrm{N}$ em resposta ao incremento da adubação, corroborando as observações apresentadas neste trabalho.

Os resultados obtidos são diferentes aos de Natale \& Marchal (2002), que em experimento com plantas cítricas de 18 meses de idade, em vasos, constataram que a adubação nitrogenada não proporcionou diferenças significativas no teor foliar de N. Porém estes autores avaliaram o N após 10 e 20 dias da aplicação do nutriente, enquanto no presente trabalho esta avaliação ocorreu após 4 meses de tratamentos.

Para Serrano et al. (2004), a incorporação de adubo formulado com liberação lenta de N, P e K (Osmocote $\left.{ }^{\circledR} 14-14-14\right)$ ao substrato aumentou de forma linear os teores foliares de $\mathrm{N}$ do porta-enxerto limoeiro 'Cravo'.

Nota-se que apenas a aplicação de fósforo resultou em incremento de $P$, com ajuste linear (Tabela 1). Bernardi et al. (2000) observaram que os teores de $\mathrm{P}$ aumentaram de $2,20 \mathrm{~g} \mathrm{~kg}^{-1}$ na dose mínima do nutriente $\left(0,19 \mathrm{~g}\right.$ planta $^{-1}$ de $\left.\mathrm{P}\right)$ até $4,42 \mathrm{e}$ $3,42 \mathrm{~g} \mathrm{~kg}^{-1}$ nas doses de 2,30 e 2,77 g de P, todavia, ainda segundo os autores, a aplicação de $\mathrm{P}$ em doses mais altas proporcionou redução nos teores deste nutriente. Já neste trabalho verifica-se um teor máximo de $1,61 \mathrm{~g} \mathrm{~kg}^{-1}$ para $\mathrm{P}$, com a dose deste nutriente de $200 \mathrm{mg}$ ou $0,2 \mathrm{~g}$.

Gallo et al. (1960) também não observaram efeito da adubação potássica sobre os teores de $\mathrm{P}$ nas folhas de laranjeira 'Baianinha'. Entretanto, 
VALE, D.W. et al. Doses de nitrogênio, fósforo e potássio...

Bernardi et al. (2000) observaram respostas à adubação potássica nos teores de $\mathrm{P}$ para laranja valência (enxertada em limoeiro 'Cravo') em experimento com NPK. A diferença de resultados entre os autores e o trabalho em questão pode ser devido às diferentes respostas à adubação entre as espécies, pois cada autor trabalhou com uma variedade específica de Citrus.

Observou-se diferença entre o fatorial e a testemunha, com resposta quadrática à aplicação de $\mathrm{N}$ e $\mathrm{P}$ no teor de $\mathrm{K}$ do porta-enxerto, sendo as doses de $\mathrm{P}$ igual a $115 \mathrm{mg} \mathrm{dm}^{-3}$ e $\mathrm{N}$ igual a $1.250 \mathrm{mg}$ $\mathrm{dm}^{-3}$ responsáveis pelo maior teor de $\mathrm{K}(13,76 \mathrm{~g}$ $\mathrm{kg}^{-1}$ ) (Tabela 1). Observa-se que as doses de $\mathrm{P}$ igual a $65 \mathrm{mg} \mathrm{dm}^{-3}$ e $\mathrm{N}$ igual a $1.310 \mathrm{mg} \mathrm{dm}^{-3}$ foram as responsáveis pelo maior teor de $\mathrm{K}$.

Serrano et al. (2004), verificaram aumento no teor de K em porta-enxerto de limoeiro 'Cravo' com a aplicação de K no substrato. Reese \& Koo (1975) também relataram que o aumento das doses de $\mathrm{K}$ resulta em aumento dos teores foliares de $\mathrm{K}$, mas não interferem nos teores de N. A aplicação de altas doses de $\mathrm{N}$ resulta em altos teores de $\mathrm{N}$ na folha e baixos teores de K (Reese \& Koo, 1975), fato não verificado no presente trabalho. Tais resultados evidenciam a interação entre teores, assim, mostrase a necessidade mais estudos elucidem o desbalanço que pode ocorrer quando se trabalha com mais de um nutriente.

Bernardi et al. (2000) observaram que o fornecimento de $\mathrm{N}$ aumentou linearmente os teores de nutrientes em mudas de citrus, porém o $\mathrm{K}$ atuou negativamente, os efeitos foram mais intensos nos teores mais elevados de $\mathrm{N}$, quando estes autores aumentaram as doses de $\mathrm{K}$ de 0,42 para $12,45 \mathrm{~g}$ planta $^{-1}$ fez com que os teores de $\mathrm{N}$ reduzissem, corroborando com os efeitos obtidos no presente trabalho.

Scivittaro et al. (2004), em experimento com porta-enxerto de limoeiro 'Cravo', em tubetes, verificaram incremento nos teores de $P$ na parte aérea com a aplicação de $\mathrm{N}$, todavia, concluíram que altas doses de $\mathrm{N}$ (acima de $0,37 \mathrm{~g} \mathrm{dm}^{-3}$ ) proporcionam redução no acúmulo de $\mathrm{P}$.

\section{Matéria seca total \\ Para massa seca total observou-se resposta significativa do fatorial $(549,01 \mathrm{mg}$ tubete $\left.^{-1}\right)$ em relação à testemunha $(251,67 \mathrm{mg}$ tubete $\left.^{-1}\right)\left(F=60,92{ }^{* *}\right)$.}

Conforme a Tabela 2 , houve resposta significativa das plantas à adubação nitrogenada e fosfatada indicando as doses de $\mathrm{N}$ igual a $1840 \mathrm{mg}$ $\mathrm{dm}^{-3}$ e de $\mathrm{P}$ igual a $90 \mathrm{mg} \mathrm{dm}^{-3}$, como as responsáveis pela maior massa seca total $(821 \mathrm{mg}$ tubete $^{-1}$ ).

TABELA 2 - Massa seca total (M.S.T.) de nutrientes do porta-enxerto limoeiro 'Cravo', aos quatro meses após a emergência, em função da adubação N, P e K.

\begin{tabular}{|c|c|c|c|c|}
\hline Variável $^{(2)}$ & Equações & Doses $^{(3)}$ & Teste $F^{(1)}$ & $Y \max .{ }^{(4)}$ \\
\hline M.S.T. NxP & $\begin{array}{l}Y=249,071+\left(4,69^{*} P\right)+\left(0,07^{*} N\right)-\left(0,016^{*} P^{*} P\right) \\
-\left(0,001^{*} P^{*} N\right)+\left(9,335 e^{-5 *} N^{*} N\right)\end{array}$ & $\begin{array}{l}\mathrm{N}=1840 \mathrm{mg} \mathrm{dm}^{-3} \\
\mathrm{P}=90 \mathrm{mg} \mathrm{dm}^{-3}\end{array}$ & $26,46^{*}$ & 821 \\
\hline M.S.T. NxK & $\begin{array}{l}Y=308,866+\left(0,075^{*} N\right)+\left(0,354^{*} K\right)+\left(3,229 e^{-5}\right. \\
\left.{ }^{*} N^{*} N\right)-\left(2,662 e^{-5 *} N^{*} K\right)-\left(1,74 e^{-4 *} K^{*} K\right)\end{array}$ & $\begin{array}{l}\mathrm{N}=1840 \mathrm{mg} \mathrm{dm}^{-3} \\
\mathrm{~K}=1015 \mathrm{mg} \mathrm{dm}^{-3}\end{array}$ & $14,41^{*}$ & 687 \\
\hline \multicolumn{5}{|c|}{$\begin{array}{l}\text { (1)** - Significativo a } 1 \% \text { de probabilidade; }{ }^{*} \text { - significativo a } 5 \% \text { de probabilidade; }{ }^{\text {ns }} \text { não significativo a } 5 \% \text { de probabilidade; } \\
\text { (2) M.S.T. NxP - massa seca da interação nitrogênio x fósforo }\left(\mathrm{g} \mathrm{kg}^{-1}\right) \text {; M. S. T. NxK - massa seca da interação nitrogênio x } \\
\text { potássio }\left(\mathrm{g} \mathrm{kg}^{-1}\right) \text {; T.K. - teor de potássio }\left(\mathrm{g} \mathrm{kg}^{-1}\right) \text {; } \\
{ }^{(3)} \text { Doses - dose do fertilizante }\left(\mathrm{mg} \mathrm{dm}^{-3} \text { de substrato) responsável pelo maior teor do nutriente; }\right. \\
{ }^{(4)} \text { Y max. - maior massa seca dos porta-enxertos de limoeiro 'Cravo' }(\mathrm{mg}) \text {. }\end{array}$} \\
\hline
\end{tabular}

Nota-se que houve interação significativa entre $\mathrm{N} \times \mathrm{P}$, sendo a dose de $\mathrm{P}$ igual a $90 \mathrm{mg} \mathrm{dm}^{-3} \mathrm{e}$ $\mathrm{N}$ igual a $1840 \mathrm{mg} \mathrm{dm}^{-3}$ estão associadas à maior massa seca total (Tabela 2). A interação significativa entre $\mathrm{N} \times \mathrm{K}$ apontou as doses de $1840 \mathrm{mg} \mathrm{dm}^{-3}$ de $\mathrm{N}$ e $1015 \mathrm{mg} \mathrm{dm}^{-3}$ de $\mathrm{K}$ como responsáveis pela maior massa seca total da parte aérea $\left(687 \mathrm{mg}\right.$ tubete $\left.{ }^{-1}\right)$. Scivittaro et al. (2004), em experimento que avaliou a resposta à adubação nitrogenada na formação do porta-enxerto de limoeiro cravo, concluíram que a máxima produção de massa seca foi obtidas com 2,47 e 4,23 g planta $^{-1}$ de $\mathrm{N}$ e K, respectivamente. Apesar dos autores terem encontrado valores superiores ao do presente trabalho para matéria seca, para $\mathrm{N}$ e $\mathrm{K}$, isto implica que talvez as doses trabalhadas pudessem ser maiores.

\section{Acúmulo de nutrientes}

Houve interação significativa $\mathrm{N} \times \mathrm{P}$ para o acúmulo de $\mathrm{N}, \mathrm{P}$ e K, e interação $\mathrm{N} x \mathrm{~K}$ para o acúmulo de $\mathrm{N}$, e interação $\mathrm{P} \times \mathrm{K}$ para acúmulo de $\mathrm{K}$ (Tabela $3)$.

Observa-se que a dose de $\mathrm{P}$ igual a $50 \mathrm{mg}$ $\mathrm{dm}^{-3}$ foi responsável pelo maior acúmulo de $\mathrm{N}$ e a dose de $\mathrm{P}$ igual a $100 \mathrm{mg} \mathrm{dm}^{-3}$ foi a responsável pelo maior acúmulo de $\mathrm{P}$ e $\mathrm{K}$ (Tabela 3 ).

Pelo desdobramento das interações $\mathrm{N}$ x $\mathrm{P}$ e $\mathrm{N} \times \mathrm{K}$, observa-se maior acúmulo de $\mathrm{N}$ com a dose de $1.840 \mathrm{mg} \mathrm{dm}^{-3}$ de $\mathrm{N}$, e com a dose de $\mathrm{P}$ igual a 50 $\mathrm{mg} \mathrm{dm}^{-3}$ (máximo de $12,85 \mathrm{mg}_{\text {planta-1}}{ }^{-1}$ ), e para dose de $\mathrm{K}$ igual a $790 \mathrm{mg} \mathrm{dm}^{-3}$ tendo maior acúmulo com 10,97 $\mathrm{mg} \mathrm{planta}^{-1}$ (Tabela 3). Bernardi et al. (2000), avaliando a resposta de citrus a adubação $\operatorname{com} \mathrm{N}, \mathrm{P}$ 
TABELA 3 - Acúmulo de nutrientes na massa seca da planta inteira do porta-enxerto limoeiro 'Cravo', aos quatro meses após a emergência, em função da adubação N, P e K.

\begin{tabular}{|c|c|c|c|c|}
\hline Variável $^{(2)}$ & Equação & $\operatorname{Doses}^{(3)}$ & Teste $\mathrm{F}^{(1)}$ & $Y \max .{ }^{(4)}$ \\
\hline A.N. & $\begin{array}{l}Y=2,479+\left(0,045^{*} P\right)+\left(6,207 e^{-4 *} N\right)-\left(1,339 e^{-4}\right. \\
\left.{ }^{*} P^{*} P\right)-\left(2,532 e^{-5 *} P^{*} N\right)+\left(2,849 e^{-6 *} N^{*} N\right)\end{array}$ & $\begin{array}{l}\mathrm{N}=1.840 \mathrm{mg} \mathrm{dm}^{-3} \\
\mathrm{P}=50 \mathrm{mg} \mathrm{dm}^{-3}\end{array}$ & $F=20,38^{*}$ & 12,85 \\
\hline A.N. & $\begin{array}{l}Y=2,884-\left(4,003 e^{-4 *} N\right)+\left(0,004^{*} K\right)+\left(2,436 e^{-6}\right. \\
\left.{ }^{*} N^{*} N\right)-\left(9,841 e^{-7 *} N^{*} K\right)-\left(1,849 e^{-6 *} K^{*} K\right)\end{array}$ & $\begin{array}{l}\mathrm{N}=1.840 \mathrm{mg} \mathrm{dm}^{-3} \\
\mathrm{~K}=790 \mathrm{mg} \mathrm{dm}^{-3}\end{array}$ & $\mathrm{~F}=21,71^{*}$ & 10,97 \\
\hline A.P. & $\begin{array}{l}Y=0,37+\left(0,008^{*} P\right)-\left(8,594 e^{-6 *} N\right)-\left(2,726 e^{-5}\right. \\
\left.{ }^{*} P^{*} P\right)-\left(1,442 e^{-6 *} P^{*} N\right)+\left(1,259 e^{-7 *} N^{*} N\right)\end{array}$ & $\begin{array}{l}\mathrm{N}=1.840 \mathrm{mg} \mathrm{dm}^{-3} \\
\mathrm{P}=100 \mathrm{mg} \mathrm{dm}^{-3}\end{array}$ & $F=10,96^{*}$ & 1,04 \\
\hline A.K. & $\begin{array}{l}Y=2,973+\left(0,054^{*} P\right)+\left(0,002^{*} N\right)-\left(2,069 e^{-4}\right. \\
\left.{ }^{*} P^{*} P\right)-\left(1,622 e^{-5 *} P^{*} N\right)+\left(5,779 e^{-7 *} N^{*} N\right)\end{array}$ & $\begin{array}{l}\mathrm{N}=1.840 \mathrm{mg} \mathrm{dm}^{-3} \\
\mathrm{P}=50 \mathrm{mg} \mathrm{dm}^{-3}\end{array}$ & $\mathrm{~F}=101,41^{* *}$ & 9,30 \\
\hline A.K. & $\begin{array}{l}Y=3,626+\left(0,063^{*} P\right)+\left(0,004^{*} K\right)-\left(2,633 e^{-4}\right. \\
\left.{ }^{*} P^{*} P\right)-\left(1,165 e^{-5 *} P^{*} K\right)-\left(1,29 e^{-6 *} K^{*} K\right)\end{array}$ & $\begin{array}{l}\mathrm{P}=100 \mathrm{mg} \mathrm{dm}^{-3} \\
\mathrm{~K}=790 \mathrm{mg} \mathrm{dm}^{-3}\end{array}$ & $F=15,96^{*}$ & 8,73 \\
\hline
\end{tabular}

(1)** - Significativo a $1 \%$ de probabilidade; * - significativo a $5 \%$ de probabilidade; ${ }^{\text {ns }}$ - não significativo a $5 \%$ de probabilidade;

${ }^{(2)}$ A.N. - acúmulo de nitrogênio ( $\left.\mathrm{mg} \mathrm{planta}^{-1}\right)$; A.P. - acúmulo de fósforo (mg planta $\left.{ }^{-1}\right)$; A.K. - acúmulo de potássio (mg planta $\left.{ }^{-1}\right)$;

${ }^{(3)}$ Doses - dose do fertilizante ( $\mathrm{mg} \mathrm{dm}^{-3}$ de substrato) responsável pelo maior acúmulo do nutriente;

(4) Y max. - acúmulo máximo dos nutrientes $\left(\mathrm{mg} \mathrm{planta}^{-1}\right)$.

e $\mathrm{K}$, constataram que as mudas de laranjeira 'Valência' enxertadas em limoeiro 'Cravo', apresentaram resposta quadrática em relação as doses de $\mathrm{N}$ aplicadas sobre os valores do nutriente nas folhas novas.

Scivittaro et al. (2004) também observaram que a aplicação de N em porta-enxerto limoeiro 'Cravo' promoveu incremento no acúmulo do $\mathrm{N}$, fato este esperado devido ter aumentado o teor e matéria seca $\mathrm{e}$, ainda, de $\mathrm{P}$ e $\mathrm{K}$, porém até certo ponto, pois o modelo que melhor se ajustou foi o quadrático, os autores citam também que o que pode ter influenciado tais dados é a interação antagônica entre os nutrientes. Ruschel et al. (2004) observaram diminuição no acúmulo de $\mathrm{N}$ com o aumento das doses de $\mathrm{P}$, corroborando com os resultados obtidos neste trabalho.

Para desdobramento da interação $\mathrm{N} \times \mathrm{P}$ observou-se que houve incremento no acúmulo de $P$. Verifica-se, ainda, que a dose de $P$ igual a $100 \mathrm{mg} \mathrm{dm}$ ${ }^{3}$ e de $\mathrm{N}$ igual a $1.840 \mathrm{mg} \mathrm{dm}^{-3}$ foram as responsáveis pelo maior acúmulo de $\mathrm{P}\left(1,04 \mathrm{mg}_{\text {planta }}{ }^{-1}\right)$ (Tabela 3$)$.

Foi encontrado também efeito entre o fatorial $\left(7,2 \mathrm{mg} \mathrm{planta}^{-1}\right)$ e a testemunha $\left(2,8 \mathrm{mg} \mathrm{planta}^{-1}\right)$, havendo resposta quadrática à adubação fosfatada, sendo a dose de $\mathrm{P}$ igual a $81 \mathrm{mg} \mathrm{dm}^{-3}$ responsável pelo maior acúmulo de $\mathrm{K}\left(\mathrm{F}=58,6^{* *}\right)$. Constatou-se efeito linear e crescente das doses de $\mathrm{N}$ no acúmulo de K.

Pelo desdobramento das interações $\mathrm{N} \times \mathrm{P}$ e $\mathrm{P} \times \mathrm{K}$, observou-se que na primeira houve incremento no acúmulo de $\mathrm{K}$ (máximo de $9,30 \mathrm{mg}$ planta $^{-1}$ ), no segundo desdobramento o máximo foi de $8,53 \mathrm{mg}$ planta $^{-1}$ ) (Tabela 3). Para a interação $\mathrm{N} \times \mathrm{P}$, as doses de $P$ igual a $50 \mathrm{mg} \mathrm{dm}^{-3}$ e $\mathrm{N}$ igual a $1.840 \mathrm{mg}$ $\mathrm{dm}^{-3}$ foram as responsáveis pelo maior acúmulo de $\mathrm{K}$. Observa-se que, para a interação $\mathrm{P} \times \mathrm{K}$, as doses de $\mathrm{P}$ igual a $100 \mathrm{mg} \mathrm{dm}^{-3}$ e de $\mathrm{K}$ igual a 790 $\mathrm{mg} \mathrm{dm}^{-3}$ foram as responsáveis também pelo maior acúmulo de $\mathrm{K}$.

Rozane et al. (2007) encontraram como melhores doses para produção de porta-enxertos de citrumelo 'Swingle' de $920 \mathrm{mg} \mathrm{dm}^{-3}$ de N, 100 $\mathrm{mg} \mathrm{dm}{ }^{-3}$ de $\mathrm{P}$ e $790 \mathrm{mg} \mathrm{dm}^{-3}$ de $\mathrm{K}$.

Segundo os dados obtidos no presente trabalho o aumento das doses em relação ao padrão de nitrogênio $\left(920 \mathrm{mg} \mathrm{dm}^{-3}\right)$ aumentou o acúmulo deste nutriente, $\mathrm{P}$ e K . Para potássio a dose padrão $\left(790 \mathrm{mg} \mathrm{dm}^{-3}\right.$ ) proporcionou bons resultados.

\section{CONCLUSÕES}

A dose de nitrogênio de $1.840 \mathrm{mg} \mathrm{dm}^{-3}$ proporcionou maior teor e acúmulo de $\mathrm{N}$ para $\mathrm{o}$ intervalo de doses estudado.

Dose de $\mathrm{K}$ de $790 \mathrm{mg} \mathrm{dm}^{-3}$ promoveu melhor estado nutricional nos porta-enxertos de limoeiro 'Cravo'.

\section{AGRADECIMENTO}

À FAPESP, pela bolsa de iniciação científica concedida ao acadêmico de Engenharia Agronômica Diego Wyllyam do Vale. 


\section{REFERÊNCIAS}

1. AGRIANUAL 2007. Anuário estatístico da agricultura brasileira. Citros. São Paulo: Instituto FNP, 2007. 504 p.

2. BARROSO, D. G.; CARNEIRO, J. G. A.; LELES, P. S. S. Qualidade de mudas de Eucalyptus camaldulensis e E. urophylla produzidas em tubetes e em blocos prensados, com diferentes substratos. Floresta e Ambiente, v. 7, n. 1, p. 238$250,2000$.

3. BERNARDI, A. C. C.; CARMELLO, Q. A. C.; CARVALHO, S. A. Desenvolvimento de mudas de citrus cultivadas em vaso em resposta à adubação NPK. Scientia Agricola, v. 57, n. 4, p. 733-738, 2000.

4. BOAVENTURA, P. S. R. Demanda por nutrientes de porta-enxertos e mudas cítricas produzidas em substrato em ambiente protegido. 2003. 63 f. Dissertação (Mestrado em Agricultura Tropical e Subtropical) Instituto Agronômico de Campinas, Campinas, 2003.

5. CARVALHO, S. A.; MATTOS JUNIOR, D.; SOUZA, M. Efeito do $\mathrm{KNO}_{3}$ nos teores de macronutrientes na massa seca total de porta-enxertos cítricos produzidos em bandejas. Bragantia, v. 59, n. 1, p. 89-94, 2000.

6. CARVALHO, S. A.; SOUZA, M. Doses e freqüência de aplicação de nitrato de potássio no crescimento do limoeiro'Cravo' e da tangerineira-'Cleópatra' em bandejas. Pesquisa Agropecuária Brasileira, v. 31, n. 11, p. 815-822, 1996.

7. CASTLE, W. S. Rootstock as a fruit quality factor in citrus and decidous tree crops. New Zealand Journal of Crop and Horticultural Science, v. 23, n. 6. p. 383-394, 1995.

8. DASBERG, S. Análises foliares de citrus em Israel. In: SEMINÁRIO INTERNACIONAL DE CITRUS-NUTRIÇÃO E ADUBAÇÃO, 4., 1996, Campinas. Anais, Campinas: Fundação Cargill, 1996. p. 41-50. (cidade do evento e numeração do evento)

9. DECARLOS NETO, A. et al. Crescimento de porta-enxertos de citrus em tubetes influenciados por doses de $\mathrm{N}$. Revista Brasileira de Fruticultura, v. 24, n.1, p. 199-203, 2002.

10. DUENHAS, L. H. et al. Produção, qualidade dos frutos e estado nutricional da laranja valência sob fertirrigação e adubação convencional. Engenharia Agrícola, v. 25, p. 154-160, 2005.

11. ESPOSTI, M. D. D.; SIQUEIRA, D. L. Doses de uréia no crescimento de porta-enxerto de citros produzidos em recipientes. Revista Brasileira de Fruticultura, v. 26, n. 1, p. 136-139, 2004.

12. ESTAT. Sistema de análises estatísticas. Jaboticabal: FCAV-UNESP, Departamento de Ciências Exatas, 1994.

13. GALLO, J. R. et al. Composição inorgânica das folhas de laranjeira Baianinha, com referência à época de amostragem e adubação química. Bragantia, v. 19, n. 3, p. 229-246, 1960.

14. LIMA, J. E. O. Novas técnicas de produção de mudas cítricas. Laranja, v. 7, n. 2, p. 463-468, 1986.

15. MALAVOLTA, E. Avaliação do estado nutricional das plantas: princípios e aplicações. Piracicaba: Potafos, 1997. p.135-198.

16. NATALE, W.; MARCHAL, J. Absorção e redistribuição de nitrogênio (15N) em Citrus mitis B11. Revista Brasileira de Fruticultura, v. 24, n. 1, p. 183-188, 2002.

17. QUAGGIO, J. A. et al. Laranjas-doce sobre diferentes porta-enxertos adubadas com nitrogênio, fósforo e potássio. Pesquisa Agropecuária Brasileira, v. 39, n. 1, p. 55-60, 2004.

18. REESE, R. L.; KOO, R. C. J. Effects of $\mathrm{N}$ and $\mathrm{K}$ fertilization on leaf analysis, tree size and yield of three major Florida orange cultivars. Journal of the American Society for Horticultural Science, v. 100, n. 1, p. 195-198, 1975.

19. ROZANE, D. E. et al. Nitrogênio, fósforo e potássio afetando a nutrição e produção de porta-enxerto de limoeiro citrumelo swingle. Revista Ceres, v. 54, n. 315, p. 422-429, 2007.

20. RUSCHEL, J. et al. Concentrações foliares do porta-enxerto limoeiro 'Cravo' em função da adubação N, P, K, Ca e S. Scientia Agricola, v. 61, n. 5, p. 501-506, 2004.

21. SAS INSTITUTE. The SAS-system for windows: release 6.11 (software). Cary: Statistical Analysis System Institute, 1996. p. 1-4.

22. SCHAFER, G. et al. Desenvolvimento vegetative inicial de porta-enxertos cítricos cultivados em diferentes sbstratos. Ciência Rural, v. 36, n. 1, p. 1723-1729, 2006.

23. SCIVITTARO, W. B. et al. Adubação nitrogenada na formação de porta-enxertos de limoeiro 'Cravo' em tubetes. Revista Brasileira de Fruticultura, v. 26, n. 1, p. 131-135, 2004.

24. SERRANO, L. A. P. et al. Efeito de sistemas de produção e doses de adubo de liberação lenta no estado nutricional de porta-enxerto cítrico. Revista Brasileira de Fruticultura, v. 26, n. 3, p. 524-528, 2004.

25. SONNEVELD, C.; ELDEREN, C. W. van. Chemical analysis of peaty growing media by means of water extraction. Communications in Soil Science and Plant Analysis, v. 25, n. 1, p. 3199-3208, 1994. 